\title{
GAIN DESIGN OF QUASI-CONTINUOUS EXPONENTIAL STABILIZING CONTROLLER FOR A NONHOLONOMIC MOBILE ROBOT
}

\author{
Shogo Nonaka ${ }^{1}$, Takeshi Tsujimura ${ }^{2}$ and Kiyotaka Izumi ${ }^{2}$ \\ ${ }^{1}$ Department of Science and Advanced Technology, Saga University, Saga, Japan \\ e-mail:12634014@edu.cc.saga-u.ac.jp \\ ${ }^{2}$ Department of Mechanical Enginnering, Saga University, Saga, Japan \\ e-mail: \{tujimura, izumi\}@me.saga-u.ac.jp
}

\begin{abstract}
The control of nonholonomic canonical form using an invariant manifold is investigated to apply to a mobile robot steered by two independent driving wheels. A quasi-continuous exponential stabilizing controller is designed by using another input pattern. Additionally, the control gain designing method is proposed for this controller. Modified error system of nonholonomic double integrator model is used as nonholonomic canonical form. Generally, the gain cannot be calculated due to the non-linear transform of system. Owing to complicated relation of several parameters, the controller behavior is inconstant by gain pattern. We propose a method of designing gain which uses desired settling time. An approximate equation to obtain designed gains is derived based on the evaluation function. The design method to determine gains of the assumed actual system is simulated. The effectiveness of the proposed method is confirmed by these simulations.
\end{abstract}

Keywords: keywords: gain design, nonholonomic canonical form, exponential stabilized control, mobile robot

Wheel type robots are widely researched as nonholonomic systems [1-3]. Among these robots, car with two independent driving wheels is easier to deal owing to fewer states and inputs. Hence, this robot is actively researched to expect wide applications. However, these robots have the restriction of non-sideslip. The restriction makes it difficult to control nonholonomic robots with simple control technique.

Nonholonomic systems cannot be stabilized by continuous feedback [4]. Nonholonomic systems are transformed into canonical form of bilinear systems. The systems enable continuous feedback control by discontinuous models or by invariant manifolds [5-9]. Using an invariant manifold enables the system to be controlled with number of input less than state one. The methods of invariant manifolds deal with phased switching control, such as attractive to an invariant manifold based on Lyapunov stability theory and continuous feedback control $[10,11]$, or discrete switching control after setting energy area based on an invariant manifold $[12,13]$. In contrast, Khennouf proposes quasicontinuous exponential stabilizing control that integrates two phase control $[14,15]$. They indicate several conditions for control design. Canonical form involves chained-form, power-form and double integrator model. We derived the unknown modified error system of the nonholonomic double integrator model, and inspected the steady-state characteristics [16]. 
In this paper, another pattern of controller about quasi-continuous exponential stabilizing control is proposed. The controller includes a control in part of attracting state variables to an invariant manifold. Additionally, we propose the method of designing gain. The gain cannot be calculated simply owing to the system non-linear transform. Owing to complicated relation of several parameter, the controller behavior is inconstant by gain pattern. We propose a method of designing gain by using desired settling time. First, eight patterns including desired values are set to remove influence of different desired values. Next, an evaluation function is set to design gain. The eight pattern simulations are evaluated by using the function with respective gain combinations. The relation of the evaluation value is verified by given initial value and gain combination to derive the approximate equation for obtaining designed gain. Finally, designed gain is simulated with the proposed method. The proposed method verifies effectiveness by comparing evaluation value.

\section{Nonholonomic Double Integrator Model}

\subsection{Kinematics of Mobile Robot}

The model of a mobile robot is shown in Figure 1 as a car with two independent driving wheels. The absolute coordinate system $O$ is set as $X$ and $Y$. Position $P$ of the robot is set the center of the axle. The distance between the left wheel and the right wheel is assigned to $2 R$. The wheel radius is assigned to $r$. The robot position is assigned to $x$ and $y$. The robot rotating angle is assigned to $\theta$. The translational velocity and angular velocity are assigned to $v$ and $\omega$, respectively.

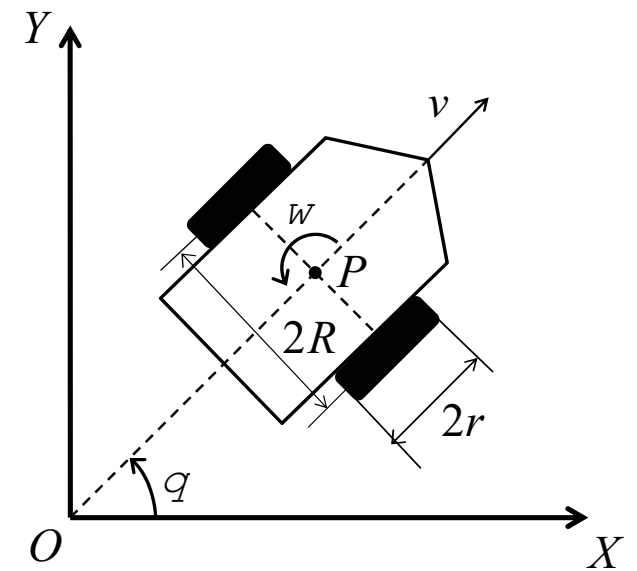

Figure 1. A nonholonomic robot platform

The state variable of the mobile robot is defined as $x(t)=[x(t) y(t) \theta(t)]^{T}$. The kinematic model is formulated as

$$
\dot{x}(t)=\left[\begin{array}{c}
\dot{x}(t) \\
\dot{y}(t) \\
\dot{\theta}(t)
\end{array}\right]=\left[\begin{array}{cc}
\cos \theta(t) & 0 \\
\sin \theta(t) & 0 \\
0 & 1
\end{array}\right]\left[\begin{array}{c}
v(t) \\
\omega(t)
\end{array}\right] .
$$

The robot cannot produce speed to the axle direction. From this condition, a nonholonomic constraint is derived as follows;

$$
\dot{x}(t) \sin \theta(t)-\dot{y}(t) \cos \theta(t)=0 .
$$

The velocity of the robot is expressed as

$$
\dot{x}(t) \cos \theta(t)+\dot{y}(t) \sin \theta(t)=v(t) .
$$

\subsection{Nonholonomic Double Integrator Model}

The conversion of non-linear state variable for $x(t)$ is

$$
z(t)=\left[\begin{array}{l}
z_{1}(t) \\
z_{2}(t) \\
z_{3}(t)
\end{array}\right]\left[\begin{array}{c}
\theta(t) \\
x(t) \cos \theta(t)+y(t) \sin \theta(t) \\
x(t) \sin \theta(t)-y(t) \cos \theta(t)
\end{array}\right] .
$$

The conversion of the state variable $z(t)$ to double integrator model is

$$
\eta(t)=\left[\begin{array}{l}
\eta_{1}(t) \\
\eta_{2}(t) \\
\eta_{3}(t)
\end{array}\right]\left[\begin{array}{c}
z_{1}(t) \\
z_{2}(t) \\
-2 z_{3}(t)+z_{1}(t) z_{2}(t)
\end{array}\right],
$$

where, the state variable is $\eta(t)$. After $\eta(t)$ is differentiated at time, robot's input conversion is defined as

$$
u(t)=\left[\begin{array}{l}
u_{1}(t) \\
u_{2}(t)
\end{array}\right]\left[\begin{array}{c}
\omega(t) \\
v(t)-z_{3}(t) \omega(t)
\end{array}\right] .
$$


From Eq.(1) to (6) we obtain

$$
\dot{\eta}(t)=\left[\begin{array}{c}
\dot{\eta}_{1}(t) \\
\dot{\eta}_{2}(t) \\
\dot{\eta}_{3}(t)
\end{array}\right]=\left[\begin{array}{c}
u_{1}(t) \\
u_{2}(t) \\
\eta_{1}(t) u_{2}(t)-\eta_{2}(t) u_{1}(t)
\end{array}\right] .
$$

\subsection{Modified Error System}

The modified error system of closed-loop system is derived. If the error system is simply set, the system does not satisfy nonholonomic double integrator model. The modified desired value must be able to derive closed-loop system that satisfies nonholonomic double integrator model. Where $x_{d}\left[\begin{array}{lll}x_{d} & y_{d} & \theta_{d}\end{array}\right]^{T}$. The transformation of non-linear state variable for $x_{d}$ is defined as

$$
z_{d}=\left[\begin{array}{l}
z_{d 1} \\
z_{d 2} \\
z_{d 3}
\end{array}\right]\left[\begin{array}{c}
\theta_{d} \\
x_{d} \cos \theta_{d}+y_{d} \sin \theta_{d} \\
x_{d} \sin \theta_{d}-y_{d} \cos \theta_{d}
\end{array}\right] .
$$

The conversion of the state variable $z_{d}$ to double integrator model is defined as

$$
\eta_{d}=\left[\begin{array}{l}
\eta_{d 1} \\
\eta_{d 2} \\
\eta_{d 3}
\end{array}\right]\left[\begin{array}{c}
z_{d 1} \\
z_{d 2} \\
-2 z_{d 3}+z_{d 1} z_{d 2}
\end{array}\right]
$$

Here, $\eta_{d}$ is the state variable. Modified desired value $\hat{\eta}_{d}(t)$ is defined as

$$
\begin{aligned}
\hat{\eta}_{d}(t) & =\left[\begin{array}{c}
\hat{\eta}_{d 1} \\
\hat{\eta}_{d 2} \\
\hat{\eta}_{d 3}(t)
\end{array}\right] \\
& {\left[\begin{array}{c}
\eta_{d 1} \\
\eta_{d 2} \\
\eta_{d 3}+\eta_{d 1}\left(\eta_{2}(t)-\eta_{d 2}\right) \\
-\eta_{d 2}\left(\eta_{1}(t)-\eta_{d 1}\right)
\end{array}\right] . }
\end{aligned}
$$

A closed-loop system satisfying nonholonomic double integrator model is constructed. The system uses the modified target value and conversion variable $\eta(t)$ of Eq. (5). The modified error $e(t)$ is expressed as

$$
e(t)=\left[\begin{array}{l}
e_{1}(t) \\
e_{2}(t) \\
e_{3}(t)
\end{array}\right]\left[\begin{array}{c}
\eta_{1}(t)-\hat{\eta}_{d 1} \\
\eta_{2}(t)-\hat{\eta}_{d 2} \\
\eta_{3}(t)-\hat{\eta}_{d 3}(t)
\end{array}\right]
$$

After $e(t)$ is differentiated by time, robot's input conversion is defined as

$$
u(t)=\left[\begin{array}{l}
u_{1}(t) \\
u_{2}(t)
\end{array}\right]\left[\begin{array}{c}
\omega(t) \\
v(t)-z_{3}(t) \omega(t)
\end{array}\right],
$$

$$
\dot{e}(t)=\left[\begin{array}{c}
\dot{e}_{1}(t) \\
\dot{e}_{2}(t) \\
\dot{e}_{3}(t)
\end{array}\right]=\left[\begin{array}{c}
u_{1}(t) \\
u_{2}(t) \\
e_{1}(t) u_{2}(t)-e_{2}(t) u_{1}(t)
\end{array}\right] .
$$

Modified error system of nonholonomic double integrator model is derived. This satisfies canonical form.

\section{Quasi-continuous Exponential Stabilizing Control}

\subsection{Invariant Manifold}

The control scheme using invariant manifold is explained as follows. We divide three variables into two parts. An invariant manifold consists of two variables as the first group. The second group consists of remaining one variable. The variables of two groups are controlled using two inputs. The control is divided into two phases per group. The first phase forms an invariant manifold using two inputs. The second phase stabilizes using two inputs while securing an invariant manifold. The flow of the control is shown in Figure 2. The invariant manifold is derived basing on a double integrator model. A control object is $e$ of Eq. (13). The issue of stabilization is handled to settle 0 in $t \rightarrow \infty$ of $e(t)$. To derive the invariant manifold, a state feedback rule of

$$
\begin{aligned}
& u_{1}(t)=-k e_{1}(t), \\
& u_{2}(t)=-k e_{2}(t),
\end{aligned}
$$

is applied to Eq. (13). Time response of closed-loop system is demanded as follows;

$$
\begin{aligned}
& e_{1}(t)=e_{1}(0) e^{-k t}, \\
& e_{2}(t)=e_{2}(0) e^{-k t} .
\end{aligned}
$$

One invariant manifold candidate can be derived as follows, using constant term $e_{3}(t)$.

$$
s(e)=e_{3}(t) \text {. }
$$

When feedback rule of Eq. (14) is applied to Eq. (13) in $k>0$, the time derivative of $s(e)$ becomes as follows;

$$
\begin{aligned}
\dot{s}(e) & =\dot{e}_{3}(t) \\
& \equiv 0 .
\end{aligned}
$$

In the feedback rule of Eq. (14), the condition of $s(e)$ is realized as

$$
s(e)=\text { Const. }
$$




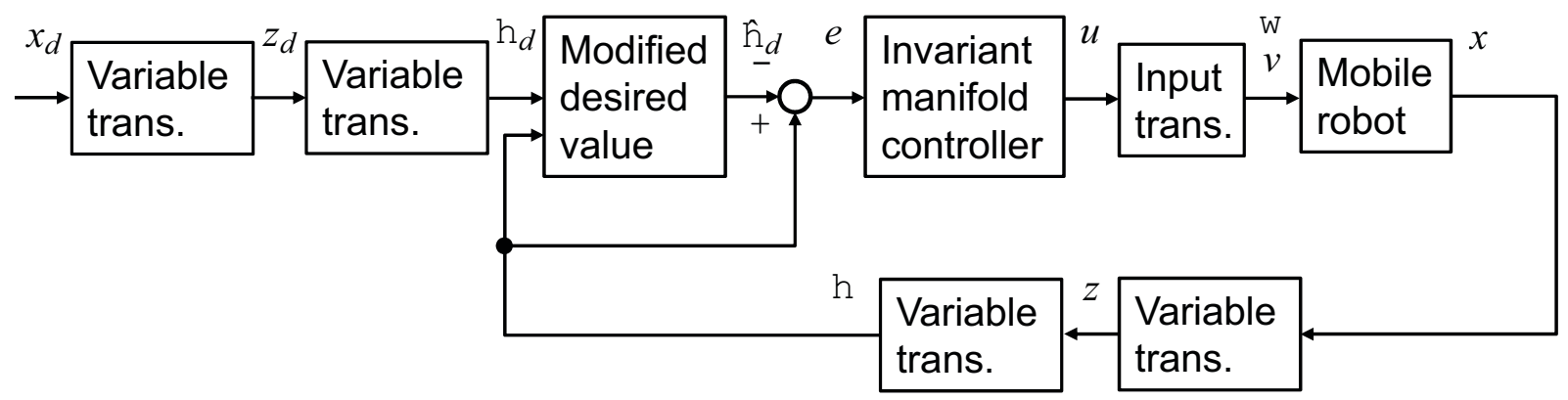

Figure 2. Feedback loop

Table 1. Desired value

\begin{tabular}{ccccccccc}
\hline$i$ & 1 & 2 & 3 & 4 & 5 & 6 & 7 & 8 \\
\hline$x_{d}$ & 7.0 & 7.0 & 7.0 & 7.0 & -5.0 & -5.0 & -5.0 & -5.0 \\
\hline$y_{d}$ & 6.0 & 6.0 & -2.0 & -2.0 & 6.0 & 6.0 & -2.0 & -2.0 \\
\hline$\theta_{d}$ & $\pi$ & 0.0 & $\pi$ & 0.0 & $\pi$ & 0.0 & $\pi$ & 0.0 \\
\hline
\end{tabular}

where, $e_{1}(t), e_{2}(t)$ become asymptotically stable. $e_{1}(t) \rightarrow 0, e_{2}(t) \rightarrow 0$ are provided at time of $t \rightarrow \infty$. Of course, $s(e)=0$, has been already satisfied. $e_{3}(t) \rightarrow 0$ is satisfied from Eq. (16).

\subsection{Stabilizing Control}

Quasi-continuous exponential stabilizing control is the integrated control. It consists of both controls of attraction to an invariant manifold, and control of convergence over an invariant manifold. In the control of two steps, controller that attracts to an invariant manifold can be set several control methods. However, there are several conditions to integrate these two controls. Design of the input must satisfy several conditions for the control. In the proposed method, single input is considered for attracting control to the invariant manifold instead of two inputs. Thus, input of $u_{1}(t)$ is set to 0 . First, the control of attraction to the invariant manifold is considered. Control rule of the first step is demanded to realize $s(e)=0$ as condition $e_{1}(0) \neq 0$ for the control of attraction to the invariant manifold. Lyapunov function of $s(e)$ is considered to be as follows;

$$
V(e)=\frac{1}{2} s^{2}(e)
$$

The control inputs are given as follows;

$$
\begin{aligned}
& u_{1}(t)=0, \\
& u_{2}(t)=-f \frac{s(e)}{e_{1}(t)} .
\end{aligned}
$$

Using $f>0$, it becomes as

$$
\begin{aligned}
\dot{V}(e) & =s(e) \dot{s}(e) \\
& =s(e)\left[e_{1}(t) u_{2}(t)-e_{2}(t) u_{1}(t)\right] \\
& =-s(e)[f s(e)] \\
& =-f s^{2}(e) \\
& \leq 0 .
\end{aligned}
$$

$\dot{V}(e)$ always becomes the negative constant at any place other than $s(e) \equiv 0 . \quad s(e) \rightarrow 0$ is provided asymptotically at time of $t \rightarrow \infty$. Therefore, input of the controller becomes as follows;

$$
\begin{aligned}
& u_{1}(t)=-k e_{1}(t), \\
& u_{2}(t)=-f \frac{s}{e_{1}(t)}-k e_{2} .
\end{aligned}
$$

Then,

$$
\begin{aligned}
\dot{s}(e)=\dot{e}_{3}(t)= & e_{1}(t) u_{2}(t)-e_{2}(t) u_{1}(t) \\
= & e_{1}(t)\left(-f \frac{s(e)}{e_{1}(t)}-k e_{2}(t)\right) \\
& -e_{2}(t)\left(-k e_{1}(t)\right) \\
= & -f s(e) .
\end{aligned}
$$


The time response is defined as

$$
s(t)=s(e(0)) e^{-f t} .
$$

It is found that, $s(t) \rightarrow 0$ in $t \rightarrow \infty$, and $e_{3}(t)$ convergences asymptotic to the origin. On the other hand, it must be as

$$
\left|\frac{s(e)}{e_{1}(t)}\right|<\infty,
$$

to keep the input of Eq. (22) to finite. $e_{1}(t)$ is asymptotically stable from Eq. (13) using Eq. (22). In addition, it becomes as

$$
\left|\frac{s(e)}{e_{1}(t)}\right|=\left|\frac{s(e(0)) e^{-f t}}{e_{1}(0) e^{-k t}}\right| \leq\left|\frac{s(e(0))}{e_{1}(0)}\right| e^{-(f-k) t},
$$

from Eq. (24). If it is satisfied as $f-k>0$, $s(e) / e_{1}(t)$ is damping exponentially in coefficient to $f-k$. That is, $e_{3}(t)$ can be converged to zero by the exponential factor of $k$, if $f \geq k$.

\section{Gain Design}

\subsection{Simulation Results Based on Evalua- tion Function}

In the derived controller, relation of gain should satisfy $f>k$. However, the controller behavior is inconstant by the given gain. Hence, the gain must be adequately designed for desired control. Nonlinearity of the system prevents us from determining proper gain easily. Thus, we investigated on the way to reduce the influence of difference of the desired values. Simulations are conducted with respect to various desired values, gains and evaluation functions. A representative evaluation value is decided regarding one combination of gains $k$ and $f$. The optimum gain combination concerning each evaluation function is determined so that the minimum evaluation-value can be provided by applying various gain combinations. Finally, an approximate equation has been derived from the selected gains and evaluation function.

First, an evaluation function is considered for one of desired value. Using desired settling time $T_{d}$, real settling time $T_{i}$ and movement distance $L_{i}$, the evaluation function for each desired values is defined by following relation.

$$
d_{i}=L_{i}+\left|T_{i}-T_{d}\right| .
$$

The evaluation of respective desired values is $d_{i}, i$ is defined as desired value number. The lower the evaluation value becomes, the more desirable the control system responds. Eight sets of desired values are defined as in Table 1 and satisfied for all relations of plus and minus with three state variables. The distance between initial position and desired position is regulated as same length because evaluation criterion contains the distance. To inspect evaluation of respective combination gain, the function evaluation $J$ is defined as follows;

$$
J=\sum_{i=1}^{8} d_{i}
$$

Eq. (27) shows evaluation variable of desired settling time $T_{d}$. Desired $T_{d}$ changes to verify gain combination for minimum evaluation value of respective $T_{d}$. An approximate equation that decides designed gain combination is derived using gain combination of respective $T_{d}$. Gain combination is inspected. Eq (26) defined gain $k$ and $f$. The initial convergence of control to the invariant manifold, and it is affected by gain $f$. Feedback control that is affected by gain $k$, must become subsequently convergence. Hence, the gain that relates the settling time is gain $k$ of finally convergence input. In this research, gain $k$ is set from 0.05 to 0.85 , in steps of 0.05 .

Next, the combination of $f$ which relates the respective combination $k$ is considered. As shown in Eq. (15) and Eq. (24), the time response exponentially relates to the respective gain. The influence of $f$ gradually becomes smaller according to the increase of $f$. On the other hand, high gain makes instability with systems. For this reason, the upper limit value of $f$ sets 90 , that is inverse of sampling time 0.01 . These conditions and prerequisite if $f>k$ are satisfied in following relation.

$$
f=a \times 10^{n},
$$

where, parameter $a$ is changed from 1 to 9 , then $n$ is changed from -1 to 1 . The gain, $f$ is assigned by combining with these respective values. Table 2 shows all gain $f$ in this case. Initial values is set to $\left[\begin{array}{lll}x & y & \theta\end{array}\right]=\left[\begin{array}{lll}1.0 & 2.0 & \pi / 2\end{array}\right]$.

Decision of convergence satisfies all conditions, which are $\left|e_{1}\right|<0.000872,\left|e_{2}\right|<0.05$ and $\left|e_{3}\right|<0.05$. 0.000872[rad] is 0.05[deg]. Five desired settling times $T_{d}$ are defined as $T_{d}[s]=$ $(10,20,30,40,50)$. Respective simulation time 
Table 2. Gain $f$

\begin{tabular}{cccccccccc}
\hline & $a=1$ & $a=2$ & $a=3$ & $a=4$ & $a=5$ & $a=6$ & $a=7$ & $a=8$ & $a=9$ \\
\hline$n=-1$ & 0.1 & 0.2 & 0.3 & 0.4 & 0.5 & 0.6 & 0.7 & 0.8 & 0.9 \\
\hline$n=0$ & 1.0 & 2.0 & 3.0 & 4.0 & 5.0 & 6.0 & 7.0 & 8.0 & 9.0 \\
\hline$n=1$ & 10.0 & 20.0 & 30.0 & 40.0 & 50.0 & 60.0 & 70.0 & 80.0 & 90.0 \\
\hline
\end{tabular}

Table 3. Simulation parameter

\begin{tabular}{cccc}
\hline Parameter & Radius of wheel & Distance between wheel & Sampling interval \\
\hline Value & $0.035[\mathrm{~m}]$ & $0.233[\mathrm{~m}]$ & $0.01[\mathrm{~s}]$ \\
\hline
\end{tabular}

$T$, is defined as $T=2 T_{d}$ [s]. Simulation parameters are shown in Table 3.

Figs. 3 to 7 show simulation results in 3D graphs about the relation of $k, f$, and evaluation value $J$ from Eq. (28). The range of $k$ obtains the range of \pm 0.10 from $k$ in minimum evaluation value of $J$. The axis of $f$ makes $\log$ scale, the range of $J$ is assigned per each $T_{d}$. Sequentially, Figure 3 shows the graph of simulation for $T_{d}=10$, Figure 4 shows for $T_{d}=20$, Figure 5 shows for $T_{d}=30$, Figure 6 shows for $T_{d}=40$ and Figure 7 shows for $T_{d}=50$. evaluate value $\left(\mathbb{T}_{d}=10[\mathrm{~S}]\right)$

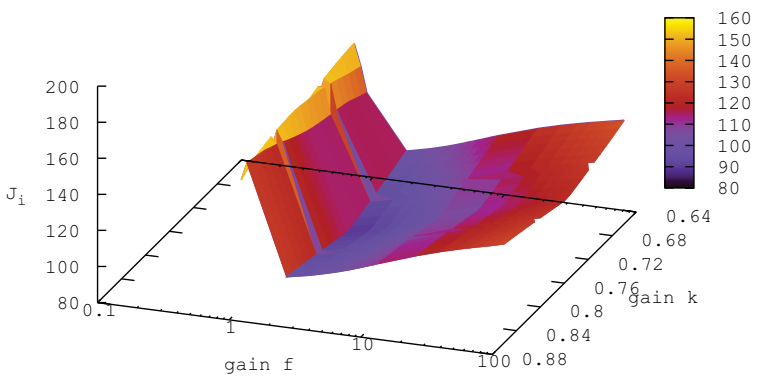

Figure 3. Evaluation value with simulation $\left(T_{d}=10\right)$ evaluate value $\left(T_{d}=20[\mathrm{~s}]\right)$

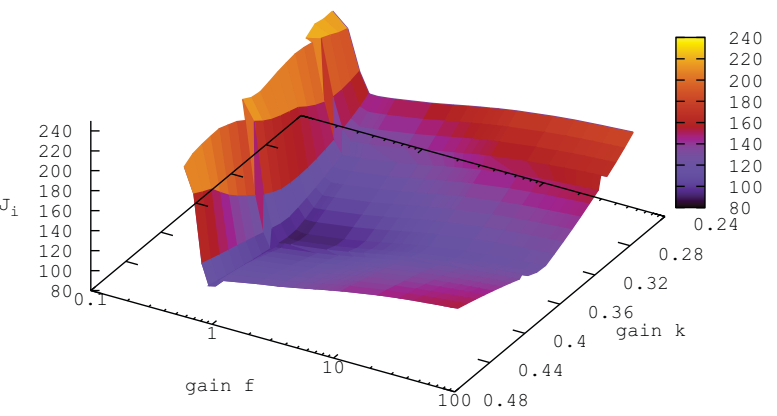

Figure 4. Evaluation value with simulation $\left(T_{d}=20\right)$

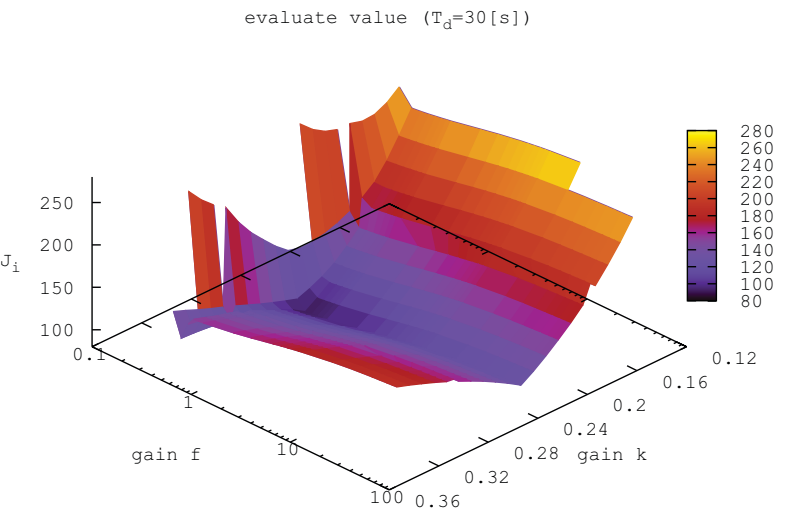

Figure 5. Evaluation value with simulation $\left(T_{d}=30\right)$ 


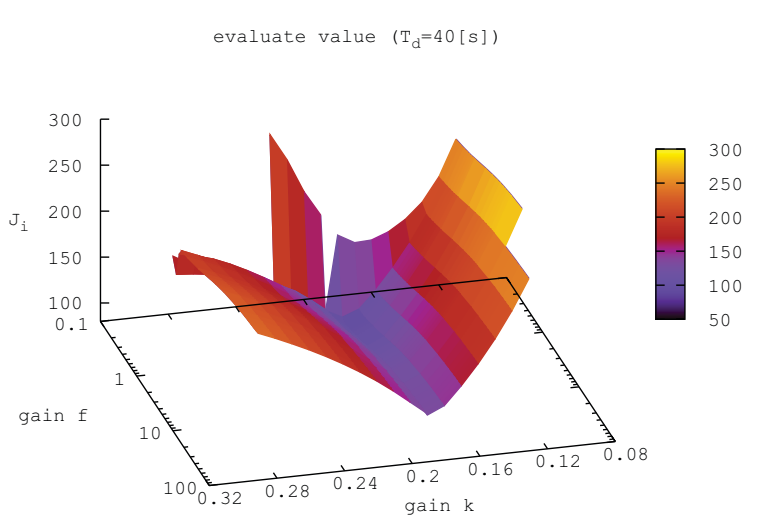

Figure 6. Evaluation value with simulation $\left(T_{d}=40\right)$

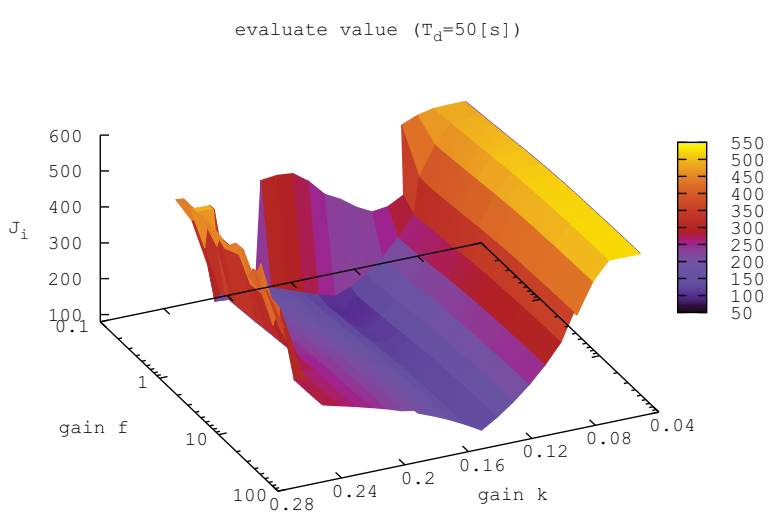

Figure 7. Evaluation value with simulation $\left(T_{d}=50\right)$

These figures show $k$ of each $T_{d}$. The evaluation value becomes low along a certain $k$. The evaluation value gradually becomes high with leaving the certain $k$. On the $k-J$ plane, the relation is shown such as a V-shaped of which bottom is located on a certain $k$. The relationship is easier to see in Figure 5, Figure 6 and Figure 7 in a relatively large $T_{d}$. Similarly, Figure 3 and Figure 4 also show the relation, however These variations are smaller than the other variations. As mentioned previously, the reason for it is believed that time response exponentially is related to gain. It should be noted that $k$ and $T_{d}$ are in inverse relationships, because, input size relates proportional to gain size as shown in Eq. (22). The gain $k$ increases with increasing $T_{d}$. In the relation of $f$, at minimum of $J$, it is about $(f \simeq 2 k)$ in all $T_{d}$ on the $f$ - $J$ plane. The gain $f$ increases with increasing the $J$ after $f \simeq 2 k$. The value of $J$ at $f<2 k$ is larger.

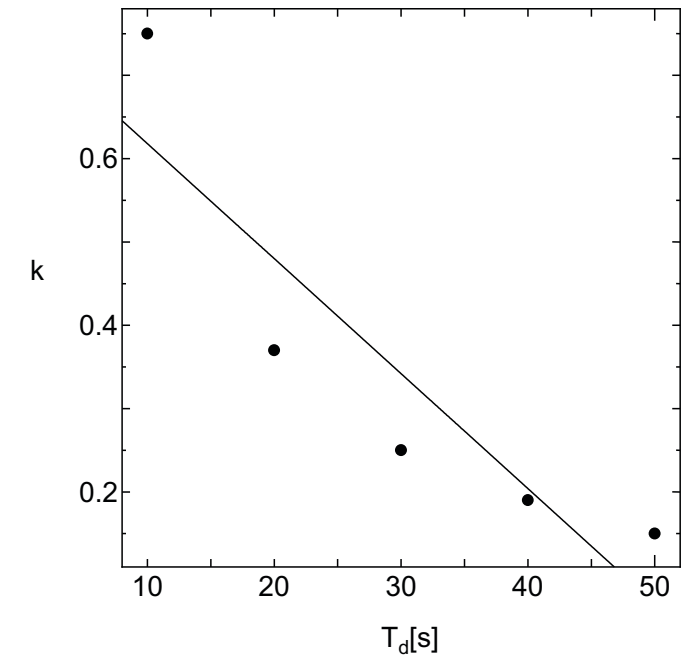

Figure 8. Approximate equation with desired settling time and gain $k$ by linear equation.

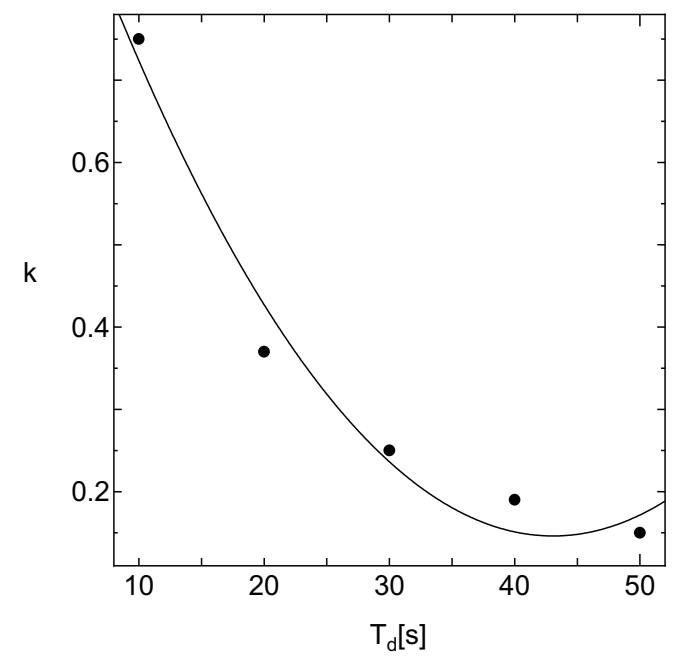

Figure 9. Approximate equation with desired settling time and gain $k$ by quadratic equation.

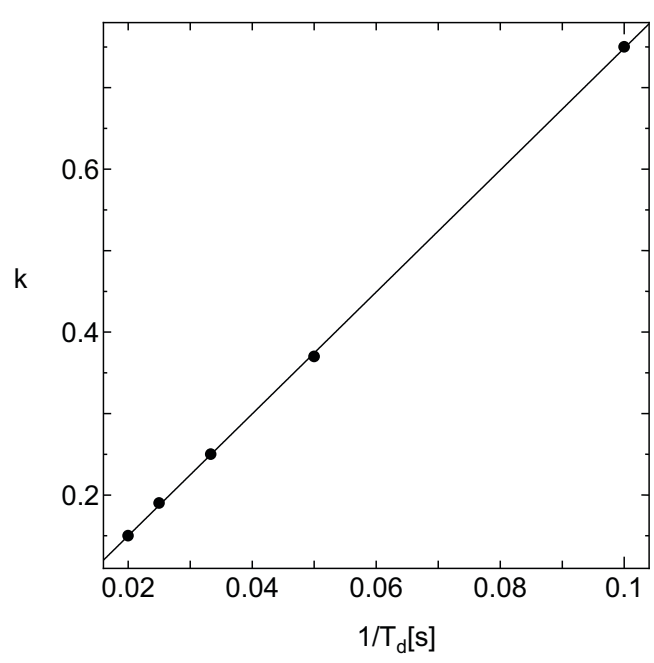

Figure 10. Approximate equation with the reciprocal of desired settling time and gain $k$ by linear equation. 


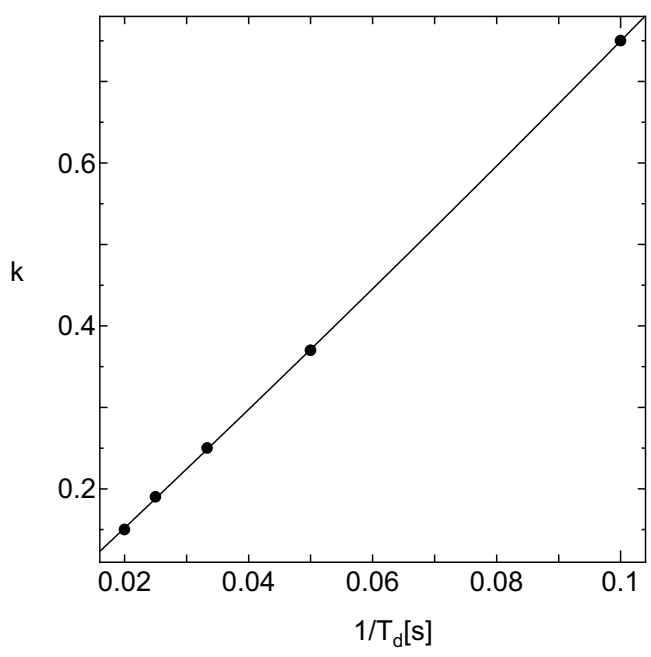

Figure 11. Approximate equation with the reciprocal of desired settling time and gain $k$ by quadratic equation.

\subsection{Design of Gain $k$}

The determination of the gain is considered by using the evaluation value of $J$ as shown in Figs. 3 7. The value of $k$ for minimum $J$ has been found to be a strong relationship with $T_{d}$. Therefore, a relation equation for deriving $k$ in minimum $J$ of respective $T_{d}$ is obtained. The relation equation of $k$ and $T_{d}$ is derived by using approximation of leastsquares method. The approximate model uses a linear equation and a quadratic equation. The applied model is decided by referring to a standard deviation $\sigma$ and a correlation coefficient $r$. The standard deviation is indexed for dispersion of data, and can indicate the irrelevant degree from approximation. The lower the value of the standard deviation becomes, the higher the accuracy of the approximate model is. The coefficient correlation is shown as interdependence degree. The correlation is higher when the value of $r$ is closer to 1 .

First, the $T_{d^{-}}-k$ graph is approximated by using linear equation as shown in Figure 8, and by using quadratic equation as shown in Figure 9. Next, the $1 / T_{d}-k$ graph is approximated by using linear equation as shown in Figure 10, and by using quadratic equation as shown in Figure 11. Each approximate equation is defined as $k=a T_{d}^{2}+b T_{d}+c$. These coefficients $a, b, c$ and the standard deviation $\sigma$ and the coefficient correlation $r$ are shown in Table 4 . The significant figure is 4 digits. $T_{d}-k$ graphs in Figure 8 and in Figure 9 show large deviation of the data and the approximate line. In contrast, the approximate line of $1 / T_{d}-k$ graph passes over the data point or passes near of data point as shown in Figure 10 and Figure 11. According to Table 4, the $\sigma$ and the $r$ prove that Figure 10 and Figure 11 are shown with higher accuracy. The difference of $\sigma$ is nearly 10 times. Next, the linear equation and the quadratic equation are compared. The $\sigma$ of the quadratic equation is smaller than the linear equation owing to high degree. However, the difference of $\sigma$ between Figure 10 and Figure 11 is small. Both correlations, $|r|$ are as high as around 1 . These values are the same to four significant figures. Hence, linear equation is sufficient to use the approximate model. Accordingly, the $k$ approximate equation is defined by Figure 10 as follows;

$$
k=7.483 \frac{1}{T_{d}}+3.040 \times 10^{-4}
$$

\subsection{Design of Gain $f$}

Here, an approximate equation for deciding $f$ by using $k$ is determined. The approximate equation of $f$ is derived by the relationships of $k$ and $f$ in minimum $J . \quad f$ is converted to $\log _{10} f$ owing the exponential relationship. The $k-\log _{10} f$ graphs are shown in Figure 12 in which approximation is based on a linear equation, and also in Figure 13 in which approximation is based on a quadratic equation.

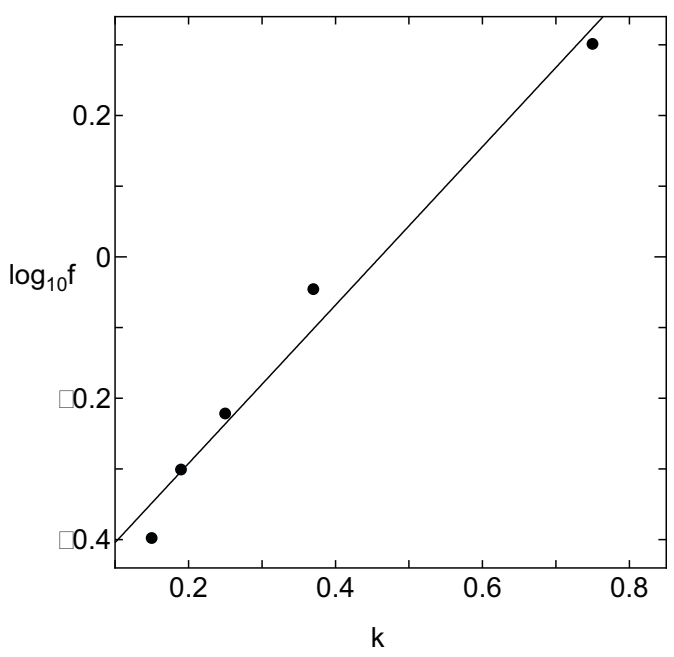

Figure 12. Approximate equation with gain $f$ and gain $k$ by linear equation. 
Table 4. Coefficient of approximate equation and approximate evaluation for gain $k$

\begin{tabular}{cccccc}
\hline Figure name & $a$ & $b$ & $c$ & $\sigma$ & $r$ \\
\hline Figure 8 & & $-1.380 \times 10^{-2}$ & $7.560 \times 10^{-1}$ & $3.084 \times 10^{-1}$ & $8.989 \times 10^{-1}$ \\
\hline Figure 9 & $5.286 \times 10^{-4}$ & $-4.551 \times 10^{-2}$ & 1.126 & $1.871 \times 10^{-1}$ & $9.869 \times 10^{-1}$ \\
\hline Figure 10 & & 7.483 & $3.040 \times 10^{-4}$ & $4.896 \times 10^{-2}$ & $9.999 \times 10^{-1}$ \\
\hline Figure 11 & 3.330 & 7.073 & $9.246 \times 10^{-3}$ & $3.834 \times 10^{-2}$ & $9.999 \times 10^{-1}$ \\
\hline
\end{tabular}

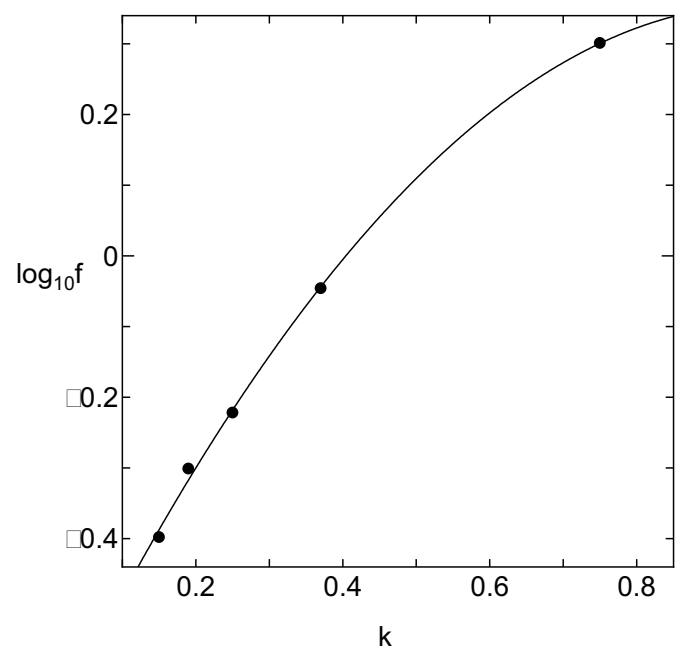

Figure 13. Approximate equation with gain $f$ and gain $k$ by quadratic equation.

Each approximate equation is defined as $k=$ $a T_{d}^{2}+b T_{d}+c$. These coefficients $a, b, c$ and the standard deviation $\sigma$ and the correlation coefficient $r$ are shown in Table 5. The significant figure is 4 digits. In a comparison between Figure 12 and Figure 13, the approximate line of Figure 13 passes nearer than of Figure 12. The standard deviation $\sigma$ of Figure 13 is also smaller than the other one. The correlation coefficient, $r$ of Figure 13 is 0.994 which is higher than the other. Accordingly, the approximate equation of gain $f$ is defined by Figure 13 as follows;

$$
\log _{10} f=-1.093 k^{2}+2.131 k-6.824 \times 10^{-1} .
$$

\section{Verification of Proposed Gain Design}

\subsection{Gain Design}

The gain is actually designed by using Eq. (30) and (31). First, $T_{d}$ is set to design. Each parameter is defined to decide $T_{d}$. In this simulation, a model of the mobile robot is made as in Table 3. The maximum velocity of the mobile robot is $0.50[\mathrm{~m} / \mathrm{s}]$. Each distance between initial position and eight desired positions is same and about $7.2[\mathrm{~m}]$. However, actual orbit rarely becomes to the shortest distance. In this case, an orbit is assumed about 10.0 [m]. Assuming that the constant velocity is $0.35[\mathrm{~m} / \mathrm{s}]$ in distance of $10.0[\mathrm{~m}]$, we have decided the settling time as 35 [s]. The reason of considering the constant velocity to $0.35[\mathrm{~m} / \mathrm{s}]$ is having the maximum velocity of the mobile robot to $0.50[\mathrm{~m} / \mathrm{s}]$. Next, gain $f$ and $k$ are designed by using $T_{d}$. In this case, the significant figure is 3 digits. $k$ is calculated by using $T_{d}$ and Eq. (30) as

$$
\begin{aligned}
k & =7.483 \frac{1}{T_{d}}+3.040 \times 10^{-4} \\
& =0.214104 \\
& \simeq 0.214
\end{aligned}
$$

where, $f$ is calculated by using $k$ and Eq. (31) as follows;

$$
\begin{aligned}
\log _{10} f & =-1.093 k^{2}+2.131 k-6.824 \times 10^{-1} \\
& =-0.050055028+0.456034-0.6824 \\
& =-0.276421028 \\
f & \simeq 0.529 .
\end{aligned}
$$

Therefore, the gains of $k$ and $f$ are decided at $T_{d}=$ 35 [s] to 0.214 and 0.529 , respectively. 
Table 5. Coefficient of approximate equation and approximate evaluation for gain $f$

\begin{tabular}{cccccc}
\hline Figure name & $a$ & $b$ & $c$ & $\sigma$ & $r$ \\
\hline Figure 12 & & 1.121 & $-5.164 \times 10^{-1}$ & $1.887 \times 10^{-1}$ & $9.895 \times 10^{-1}$ \\
\hline Figure 13 & -1.093 & 2.131 & $-6.824 \times 10^{-1}$ & $9.374 \times 10^{-2}$ & $9.994 \times 10^{-1}$ \\
\hline
\end{tabular}

\subsection{Simulation with Designed Gain}

The calculated gain is simulated. The simulation parameter is used by Table 3. The evaluation value consists of Eq. (27) and Eq. (28). The initial value is $\left[\begin{array}{lll}x & y & \theta\end{array}\right]=\left[\begin{array}{lll}1.0 & 2.0 \pi / 2\end{array}\right]$. The eight desired values are defined by Table 1 . In this case, Table 6 shows the evaluation value.

Table 6. Evaluation value of simulation with the designed gain

\begin{tabular}{cccc}
\hline$T_{d}$ & $k$ & $f$ & $J$ \\
\hline 35 & 0.214 & 0.529 & 87.417 \\
\hline
\end{tabular}

To verify effectiveness of the gain designing method, Table 7 shows gain combinations in which $J$ becomes the minimum value at each $T_{d}$.

Table 7. Evaluation minimum value of simulation each desired settling time

\begin{tabular}{cccc}
\hline$T_{d}$ & $k$ & $f$ & $J$ \\
\hline 10 & 0.75 & 2.0 & 88.175 \\
\hline 20 & 0.37 & 0.9 & 89.175 \\
\hline 30 & 0.25 & 0.6 & 87.664 \\
\hline 40 & 0.19 & 0.5 & 92.403 \\
\hline 50 & 0.15 & 0.4 & 88.319 \\
\hline
\end{tabular}

The minimum $J$ fluctuates to $92.4 \lesssim J \lesssim 87.6$ as shown in Table 7. The average is $\bar{J} \simeq 89.1$. When the values $J$ of Table 7 and Table 6 are compared, the value $J$ that is simulated by using designed gain is the lowest in the values of Table 7 . Hence, the gain that is designed by this method is confirmed. As a result, the simulation is conducted under the condition that initial value $\left[\begin{array}{lll}x & y & \theta\end{array}\right]$ is $\left[\begin{array}{lll}1.0 & 2.0 & \pi / 2\end{array}\right]$

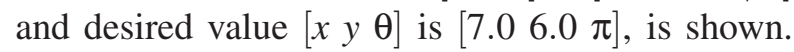
Figure 14 shows a response of modified error $e$. Figure 15 shows the orbit of the mobile robot. Figure 16 shows a response of input $u$. Figure 17 shows the robot's speed of translation $v$ and angular velocity $\omega$. Figure 14 shows a convergence to 0 in all the state $e$. Robot's state is set to desired state as shown in Figure 15. The orbit is changed by desired value. In this simulation, movement distance becomes about $13.9[\mathrm{~m}]$ by occurrence of turning over. In another pattern, movement distance becomes about 7.9 [m]. The average movement distance of the eight desired values is 10.9 [m]. Therefore, It is enough to estimate that the movement distance is 1.5 times more than the shortest distance. Figure 16 shows convergence of input to 0 according to the state. Figure 17 shows the outputs that are the translational velocity and the angular velocity according to the input. 


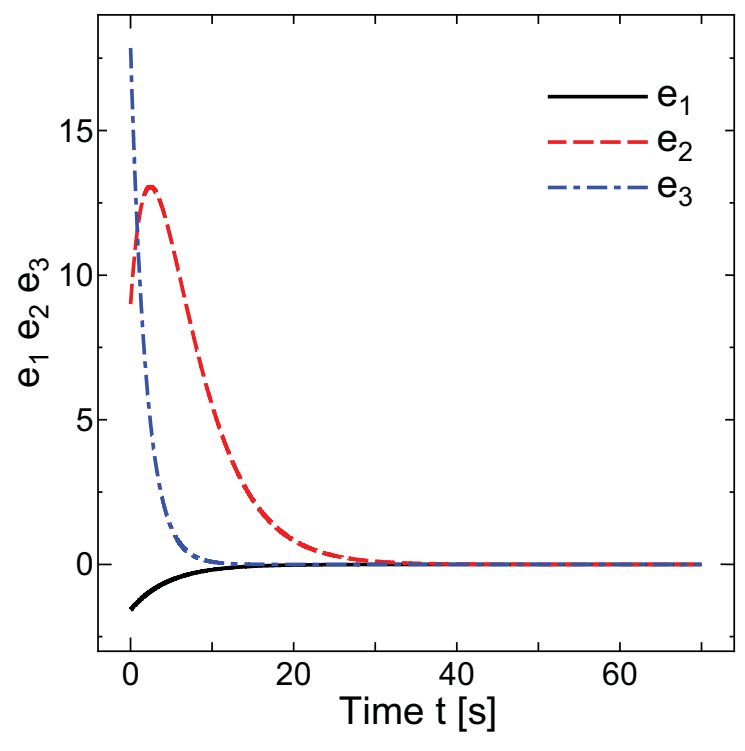

Figure 14. Error of state with canonical form

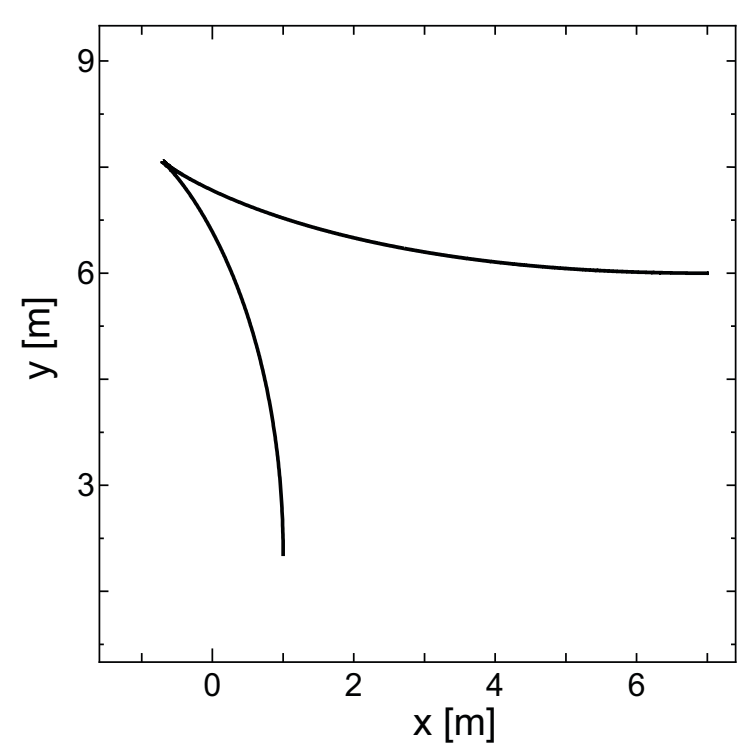

Figure 15. Orbit of mobile robot

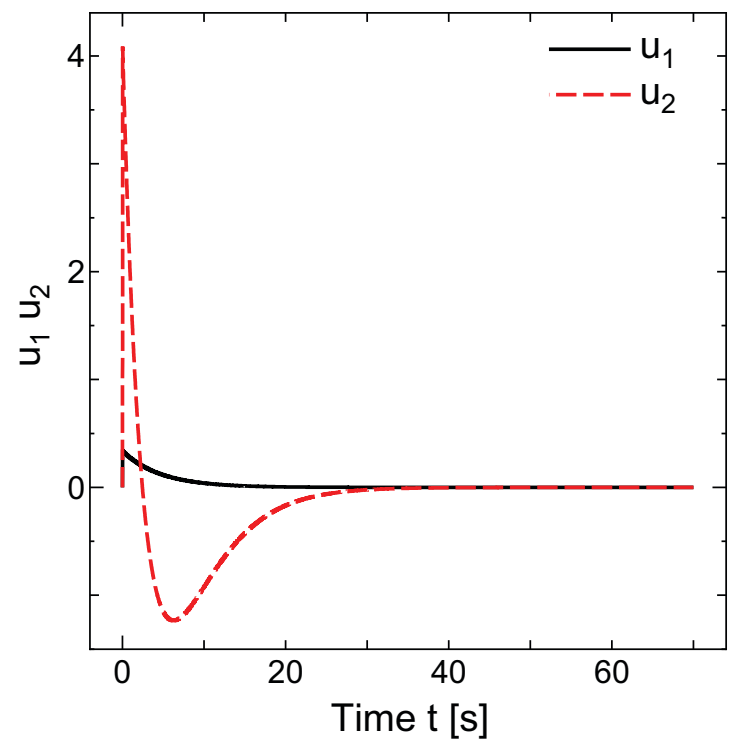

Figure 16. Inputs

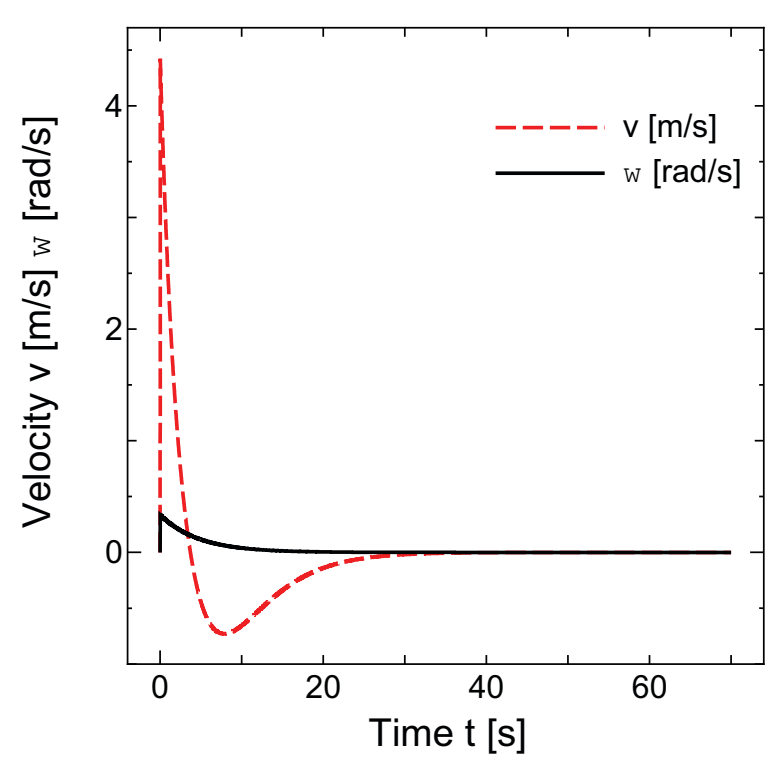

Figure 17. Relationship between time and velocity 
The maximum velocity is larger than about $0.50[\mathrm{~m} / \mathrm{s}]$ owing to the initial state value that is larger than the later state. The solution of the problem needs input saturated measures. The average velocity of the simulation is about $0.40[\mathrm{~m} / \mathrm{s}]$. The velocity is larger than the assumed velocity owing to the movement distance that becomes longer by behavior of controller. For example, there is another simulation in which maximum velocity is about $0.80[\mathrm{~m} / \mathrm{s}]$, and in which average velocity is about $0.23[\mathrm{~m} / \mathrm{s}]$. The average velocity of eight simulations becomes about $0.31[\mathrm{~m} / \mathrm{s}]$. This is close to the assumed velocity $0.35[\mathrm{~m} / \mathrm{s}]$ because it is $88 \%$ of the velocity. These results prove the effectiveness of gain that is obtained by using proposed designing method.

\section{Conclusions}

In this paper, our approach is verified by a quasi-continuous exponential stabilizing controller using another pattern of input that is the control of attractive to the invariant manifold. A quasicontinuous exponential stabilizing controller consists of a continuous feedback control and a control of attractive an invariant manifold. We focused on the control method to attract an invariant manifold. In accordance with the method of Khennouf, the control of attractive to the invariant manifold consists of single input. The modified error system of a nonholonomic double integrator model is used in this research as a nonholonomic canonical forms. This method is expected to be applied to other nonholonomic canonical forms.

We verified the stabilization of the derived quasi-continuous exponential stabilizing controller. Furthermore, we proposed the designing method of control gain by the controller. In order to design the control gain, evaluation function for simulation at each gain is defined. The evaluation function is defined by desired settling time, real settling time and movement distance. Additionally, the eight patterns of desired values are constituted for satisfying all relations of plus and minus with three state variables to consider the influence from initial value. The approximate equation is derived from the evaluation value. Derivation of gain $k$ has been approximated by linear equation using a relationship between $k$ and $1 / T_{d}$ which is the inverse of desired settling time $T_{d}$. Derivation of gain $f$ is approximated by quadratic equation using a relationship between $\log _{10} f$ and $k$. Accordingly, it is possible to determine the gains $k$ and $f$ by giving the desired settling time $T_{d}$ with the method.

This design method that determines the gain of the assumed actual system has been simulated. The simulation was verified by using the evaluation function. The evaluation value of the simulation using designed gain with the method has become the better value than several evaluation values of simulation without the proposed method. The average movement distance of the eight desired values is $10.9[\mathrm{~m}]$. The average velocity of eight simulations becomes about $0.31[\mathrm{~m} / \mathrm{s}]$. By contrast, assumed movement distance is 10 [m]. Assumed average velocity is $0.35[\mathrm{~m} / \mathrm{s}]$. These relations are obtained high correlative as nearly to $90 \%$. Therefore, the effectiveness of the proposed method has been verified by these results.

\section{References}

[1] T.-C. Lee, Exponential stabilization for nonlinear systems with applications to nonholonomic systems, Automatica, vol. 39, no. 6, pp. 1045-1051, 2003.

[2] A. Bloch, S. Drakunov, Stabilization and tracking in the nonholonomic integrator via sliding modes, Systems and Control Letters, vol. 29, no. 2, pp. 9199, 1996.

[3] G. Escobar, M. Reyhanglu, Regulation and tracking of the nonholonomic double integrator: A fieldoriented control approach, Automatica, vol. 34, no. 1, pp. 125-131, 1998.

[4] R. W. Brockett, Asymptotic stability and feedback stabilization, Differential Geometric Control Theory, pp. 181-191, 1983.

[5] J. Luo, P. Tsiotras, Control design for chained-form systems with bounded inputs, System and Control Letters, vol. 39, no. 2, pp. 123-131, 2000.

[6] Z. Sun, S.S. Ge, W. Huo, T.H. Lee, Stabilization of nonholonomic chained systems via nonregular feedback linearization, System and Control Letters, vol. 44, no. 4, pp. 279-289, 2001.

[7] N. Marchand, M. Alamir, Discontinuous exponential stabilization of chained form systems, Automatica, vol. 39, no. 2, pp. 343-348, 2003.

[8] K. Cao, Global -exponential tracking control of nonholonomic systems in chained-form by output feedback, Acta Automatica Sinica, vol. 35, no. 5, pp. 568-576, 2009. 
[9] O. J. Sordalen, O. Egeland, Exponential stabilization of nonholonomic chained systems, IEEE Trans. on Automatica Control, vol. 40, no. 1, pp. 35-49, 1995.

[10] A. Astolfi, Discontinuous control of nonholonomic systems, Systems and Control Letters, vol. 27, no. 1 , pp. 37-45, 1996.

[11] A. Astolfi, Discontinuous control of the brockett integrator, Proc. of the 36th IEEE Conference on Decision and Control, vol. 5, pp. 4334-4339, 1997.

[12] J. P. Hespanha, A. S. Morse, Stabilization of nonholonomic integrators via logic-based switching, Automatica, vol. 35, no. 3, pp. 385-393, 1999.

[13] R. N. Banavar, Switched control strategies for underactuated Systems, Manuscripts of Invited talk, ACODS07, 2007.
[14] H. Khennouf and C. Canudas de Wit, On the construction of stabilizing discontinuous controllers for nonholonomic systems, Proc. of IFAC Nonlinear Control Systems Design, pp. 747-752, 1995.

[15] H. Khennouf, C. Canudas de Wit, Quasicontinuous exponential stabilizers for nonholonomic systems, Proc. of IFAC 13th Triennial World Congress, pp. 49-54, 1996.

[16] S. Nonaka, T. Tsujimura and K. Izumi: Modified error system of nonholonomic double integrator model using invariant manifold control, Proceedings of SICE Annual Conference 2014, pp. 42-47, 2014.

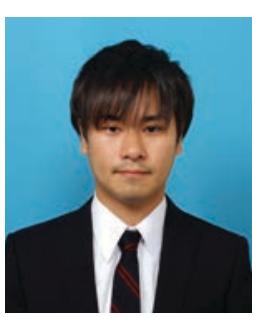

Shogo Nonaka, he received his B.E. and M.E. degrees from SAGA University, Japan, in 2006 and 2012, respectively. He is currently a Ph.D. student at SAGA University. His research interests include control systems design.

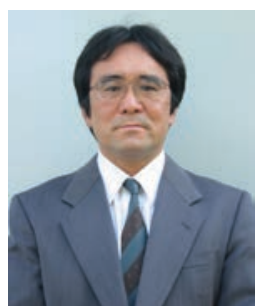

Takeshi Tsujimura, he received the B.S. and M.S. and the Ph.D. degrees in precision mechanical engineering from Tokyo University in 1981, 1983 and 1995, respectively. He was a senior research engineer of Access Network Service Systems Laboratories, Nippon Telegraph and Telephone Corporation, and worked as a professor of University of Tsukuba, Japan. Now, he serves at Saga University, Japan as a professor of Department of Mechanical Engineering. He received Young Engineer Award of the Japan Society of Mechanical Engineers in 1989, Best Paper Award of SICEICCAS in 2006 and Best Paper Award of OPTICS/ICETE in 2014.

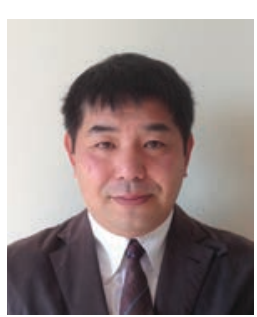

Kiyotaka Izumi received B.E. degree in Electronic Engineering from the Nagasaki Institute of Applied Science in 1991, M.E. degree in Electrical Engineering from the Saga University in 1993, and D.E. degree in Faculty of Engineering Systems and Technology from the Saga University in 1996. From 1996 to 2004, he was a Research Associate in the Department of Mechanical Engineering at Saga University. From 2004, he was an Associate Professor of Saga University. His research interests are: nonlinear control, softcomputing, man-machine interface, intelligent control. 